\title{
Haematological Manifestations of Inborn Errors of Metabolism
}

\section{Melis $\operatorname{Kose}^{1}$ and Yeșim Oymak ${ }^{2}$}

${ }^{1}$ Izmir Katip Celebi University Medical Faculty, Department of Pediatrics, Division of Pediatric Metabolism and Nutrition, Izmir, Turkey

${ }^{2}$ University of Health Sciences, Behçet Uz Children Training and Research Hospital, Department of Pediatrics, Division of Hematology, Izmir, Turkey

*Corresponding Author: Melis Kose, Izmir Katip Çelebi University Medical Faculty, Department of Pediatrics, Division of Pediatric Metabolism and Nutrition, Izmir, Turkey.

DOI: 10.31080/ASPE.2021.04.0332
Received: November 13, 2020

Published: December 10, 2020

(C) All rights are reserved by Melis Kose and Yeşim Oymak.

\begin{abstract}
Introduction: Multisystemic involvement of inborn errors of metabolism (IEMs) makes treatment and follow-up of these diseases difficult. Haematological manifestations are significantly important for both treatment and follow-up processes of IEMs. This study evaluated haematological manifestations that may coexist with IEM disorders and affect the diagnosis process to raise awareness of IEMs coexisting with haematological findings.

Materials and Methods: In this study, medical records of 132 patients diagnosed with IEM were examined retrospectively, and the data of 43 patients with haematological findings at the time of diagnosis were evaluated. The information assessed included demographic findings, diagnosis, age at diagnosis, follow-up period, haematological manifestation, and coexisting findings.

Results: An evaluation of the haematological abnormalities at the time of diagnosis showed that of the 43 patients 15 (33.3\%) had neutropenia, 3 (7.1\%) had pancytopenia, 15 (34.3\%) had iron deficiency anaemia (IDA), 15 (35.7\%) had thrombocytopenia, 7 (16.6\%) had coagulation abnormalities, 1 (2.3\%) had hemolytic anaemia, 1 (2.3\%) had hemophagocytic lymphohistiocytosis (HLH), and 7 (16.6\%) had coagulation abnormalities. Overall, 15 (35.7\%) patients had chronic anaemia.

Conclusion: The results show that hematologic abnormalities are very common features of IEM. The findings are valuable for raising awareness of hematologic manifestations in these disorders.
\end{abstract}

Keywords: Inborn Errors of Metabolism; Anemia; Neutropenia

\section{Introduction}

Inborn errors of metabolism (IEMs) are diseases that are mostly inherited and very rarely de novo genetic due to defects that occur during the breakdown or biosynthesis of certain substances at specific metabolic pathways [1]. Haematological manifestations appear as symptoms associated with many different mechanisms due to the nature of the disease, as well as IEM, being a chronic disease [2].

As consanguineous marriages have been more common in Turkey than Western societies, IEMs that are mostly inherited in an autosomal recessive pattern are more common than expected
$[3,4]$. One of the major problems in diagnosing IEMs is ruling out multisystemic problems that might be associated with a metabolic disease. Hence, many individuals with IEMs cannot benefit from treatment options or maybe late to benefit from treatment. A significant portion of IEM diseases demonstrates haematological abnormalities $[2,5,6]$. Because patients are first admitted to paediatrics and internal medicine departments before diagnosis, raising awareness about IEM coexisting with haematological findings is very important for a timely diagnosis. This study evaluated the haematological findings of patients who were diagnosed at our centre and had haematological findings at the time of diagnosis. 


\section{Materials and Methods}

The medical records of 137 patients diagnosed with IEMs at the Department of Metabolism between February 2015 and September 2019 were examined retrospectively, and 43 patients with haematological findings at the time of diagnosis were included in the study. Patients with haematological findings during follow-up were excluded from the study. The patients were divided into three groups: group 1 (intoxication-type metabolic diseases), group 2 (energy metabolism disorders), and group 3 (complex molecular disorders). The following characteristics were recorded: patient age, sex, ethnic origin, diagnosis, age at diagnosis, age at hematologic assessment, follow-up period, haematological abnormalities at the time of diagnosis, classification of metabolic disease, presence of metabolic acidosis or lactic acidosis, ammonia levels, number of IEM attacks per year presenting with decompensation, presence of growth and developmental retardation, presence of neurological symptoms, complete blood count results, presence of cytopenia, bleeding time test results (if any), B12-folic acid levels, haematological manifestation, bone marrow findings, presence of organomegaly, bleeding symptoms, and whether there was an improvement in symptoms and parameters.

\section{Results}

This study examined the data of 43 patients with haematological findings at the time of diagnosis and presentation who were diagnosed with IEMs. The female/male ratio was $23 / 20$, the age at diagnosis ranges between 17 days and 16 years (average: $38.6 \pm$ 48.73 months), maximum follow-up time was 48 months (average: $16.88 \pm 11.15$ months). When the patients were evaluated by IEM group, 15 (36.5\%) patients were included in group 1, 10 (23.8\%) in group 2, and 17 (40.2\%) in group 3. Lysinuric protein intolerance (LPI) was present in 1 patient, propionic aciduria (PA) in 3, methylmalonic aciduria (MMA) in 4, argininosuccinic aciduria in 1, citrullinemia type I in 1, ornithine transcarbamylase 1 deficiency (OTC1D) in 1, abetalipoproteinemia in 2, glycogen type I (GSD [2 GSD Ib and 1 GSDIa]) in 3, Pompe disease (PD) in 1, Gaucher disease ([GD] 8 type I, 2 types II, and 3 types III) in 13, congenital glycosylation disorders (1 ALG1-CDG, 1 DOLK-CDG, and 1 MPI-CDG) in 3 , cobalamin $\mathrm{C}$ defect (CblCD) in 1 , and mitochondrial disease (1 FBXL4-related mtDNA depletion syndrome; 1 pyruvate dehydrogenase deficiency [PDHD], and 2 Barth syndrome) in 4. An evaluation of the patients' hematological abnormalities at the time of diagnosis found that $15(33.3 \%)$ had neutropenia, 3 (7.1\%) had pancytopenia, 15 (34.3\%) had iron deficiency anemia (IDA), 15 (35.7\%) had thrombocytopenia, 7 (16.6\%) had coagulation abnormalities, $1(2.3 \%)$ had hemolytic anemia, 1 (2.3\%) had HLH, 7 (16.6\%) had coagulation abnormalities, and 15 (35.7\%) had chronic anemia. The minimum absolute neutrophil count (ANC) in the patients diagnosed with Barth syndrome was $1.1 \times 10^{9} /$ l. Neutropenia in patients with IVA, PA, and MMA was not cyclical. In the patients diagnosed with Barth syndrome, the interval between neutropenia attacks ranged from 28 to 42 days. Nine patients had concomitant hepatomegaly (HMG), and 14 had concomitant hepatosplenomegaly (HSMG). Malnutrition was detected in 23 (54.7\%) patients.

\section{Discussion}

Haematological findings are often encountered during the course of IEMs. The identification of haematological findings is very important for the diagnosis and follow-up of IEM. Some of these findings arise from the nature of the disease, while some occur due to dietary changes, malnutrition, frequent hospitalization, and other factors. Furthermore, they occasionally change the course of diseases. Some of the manifestations caused by the nature of the disease may be improved by specific treatment for the disease. Thus, the haematological findings determined in the diagnosis and presentation of IEM were investigated in this study.

Thrombocytopenia $(15 ; 35.7 \%)$ was the most commonly detected condition in our study, followed by neutropenia $(14 ; 33.3 \%)$ by a narrow margin. Cytopenia was detected especially in patients with organic aciduria and Gaucher disease. For MMA, PA, and IVA, which we identified as ketotic hyperglycinemia based on a study conducted by Inoue., et al. in which ketoacidosis-related bone marrow hypoplasia was first identified in a patient with MMA, the etiopathogenesis of pancytopenia was demonstrated to be associated with bone marrow inhibition. Cytopenia can be primarily defined as inhibition of bone marrow precursors by CoA esters [7]. However, bone marrow inhibition occurs as a result of accumulating free organic acids and metabolites in other intermediary pathways as well as due to multiple systemic effects of mitochondrial dysfunction caused by increased oxidative stress [8,9]. In our study, cytopenia occurred in patients with organic aciduria during attacks. This was highly compatible with a study showing that bone marrow cells stopped growing with the addition of methylmalonic acid into bone marrow cultures from MMA patients [7]. One of the neutropenia patients (P37) was diagnosed with mitochondrial DNA depletion. The patient had neutropenia that was non-continuous, recurrent, and mostly accompanied by an infection that took 
longer than organic aciduria and was independent of lactic acidosis attacks. A literature search found that neutropenia was detected in 16\% of FBXL4-related mitochondrial DNA depletion syndrome cases. However, the relationship between neutropenia and lactic acidosis has not yet been elucidated [10-13]. Among patients with neutropenia, another group that needs special attention is Barth syndrome and methylglutaconic aciduria. Patients with Barth syndrome are expected to have an absolute neutrophil count (ANC) below $0.5 \times 10^{9} / 1$ and a cycle length ranging from 21 to 29 days $[14,15]$. Previously identified known pathogenic variants in the TAZ gene were determined in both of our Barth syndrome patients. Although one was followed up for 6 months and the other for 12 months, their ANC never declined below $0.5 \times 10^{9} / \mathrm{l}$. The lowest ANC was $1.1 \times 10^{9} /$ l. In our study, the 2 patients with Barth syndrome and those with methylglutaconic aciduria type VII (MGCA VII) had methylglutaconic acid excretion. The patient with MGCA VII (CLPB gene defect-related MGCA VII) also had cataracts, apparent growth retardation, microcephalia, and urinary excretion of methylglutaconic acid. Their ANC levels ranged from 1000 to 1500 x $10^{9} /$ l. Although very few cases have been reported in the literature, severe neutropenia is likely to be life-threatening. However, our patient had no severe neurological findings and neither severe neutropenia nor serious infection was observed. In patients with neurological findings, the presence of neutropenia should suggest IEMs, especially Barth syndrome and CLPB-related MGCA VII.

Another disease group with a strong relationship between cytopenia and IEM is Gaucher disease. All 13 Gaucher patients in this study had thrombocytopenia, while 3 had combined thrombocytopenia and neutropenia. The Gaucher patients' ANC levels range between $0.45-7.810^{9} /$ l. Because of the early loss of P24 and P25, their ANC levels could not be monitored. However, the ANC level of P23 $\left(0.7 \times 10^{9} / \mathrm{l}\right.$ at the time of diagnosis) reached $1.5 \times 10^{9} / \mathrm{l}$ after 6 weeks from ERT $60 \mathrm{IU} / \mathrm{kg} / \mathrm{EOW}$, still within normal limits.

In our study, IDA was detected in 14 patients in 7 different disease groups (Barth syndrome, LPI, OTC1D, abetalipoproteinemia, GSD Ib, Gaucher disease, MPI-CDG, and PDH deficiency). The coexistence of an iron deficiency with IEM often tends to be associated with a restricted diet $[5,6]$. However, the patients in this study might have had IDA at the time of diagnosis. Among them, those with OTC1D and LPI refused to eat protein food and thus had a vegan-like diet. A behavioural change after eating protein foods was described, but no decompensation attacks were noted by the fam- ily. In particular, these two patients emphasized the importance of dietary intake for the differential diagnosis of IDA detected in the infantile and juvenile periods. The fact that $7 / 14$ patients with IDA had malnutrition also explains the high rate of IDA.

Three patients diagnosed with CDG had coagulation disorders at the time of diagnosis. However, none had a bleeding disorder that causes symptoms. Changes in the levels of protein $C, S$, and ATIII, which are glycosylated proteins synthesized in the liver, are known to induce changes in coagulation parameters. The patients other than the MPI-CDG patients usually had no bleeding findings that caused symptoms. Our patients' results were compatible with the symptoms [16].

\section{Conclusion}

This study evaluated the longitudinal data of IEM patients from two referral centres. We also examined the global literature on hematologic abnormalities in IEM. The results demonstrated that hematologic abnormalities are very common features of IEM. The findings are valuable for raising awareness of hematologic manifestations in these disorders.

\section{Competing Interest Statement}

Melis Kose and Yeșim Oymak, declare that they have no conflict of interest.

\section{Details of Ethics Approval}

The study was approved by the local ethics committee at the coordinating center (Katip Çelebi University Faculty of Medicine) and by all clinical partners. The current publication project was evaluated by the scientific board.

\section{Ethics Approval}

All the procedures followed were in accordance with the ethical standards of the responsible committee on human experimentation (institutional and national) and with the Helsinki Declaration of 1975, as revised in 2000. Informed consent with regard to being included in the study was obtained from all patients or their legal guardians prior to being included in the study in countries where this was needed by law.

\section{Bibliography}

1. Morava E., et al. "Quo vadis: the re-definition of "inborn metabolic diseases". Journal of Inherited Metabolic Disease 38.6 (2015): 1003-1006.

Citation: Melis Kose1 and Yeșim Oymak. "Haematological Manifestations of Inborn Errors of Metabolism”. Acta Scientific Paediatrics 4.1 (2021): $02-05$. 
2. Evangeliou A., et al. "Hematological abnormalities in inborn errors of metabolism--how frequent are they? The Cretan experience". Pediatric Hematology and Oncology 19.8 (2002): 581-585.

3. Yıldız Y., et al. "Genotypes and estimated prevalence of phosphomannomutase 2 deficiency in Turkey differ significantly from those in Europe". American Journal of Medical Genetics Part A 182.4 (2020): 705-712.

4. Koc I. "Prevalence and sociodemographic correlates of consanguineous marriages in Turkey". Journal of Biosocial Science 40.1 (2008): 137-148.

5. Tavil B., et al. "Haematological findings in children with inborn errors of metabolism”. Journal of Inherited Metabolic Disease 29.5 (2006): 607-611.

6. Sal E., et al. "Hematologic Findings of Inherited Metabolic Disease: They are More Than Expected". ournal of Pediatric Hematology/Oncology 40.5 (2018): 355-359.

7. Inoue S., et al. "Inhibition of bone marrow stem cell growth in vitro by methylmalonic acid: a mechanism for pancytopenia in a patient with methylmalonic acidemia". Pediatric Research 15.2 (1981): 95-98.

8. Chandler RJ., et al. "Mitochondrial dysfunction in mut methylmalonic acidemia”. FASEB Journal 23.4 (2009): 1252-1261.

9. Kölker S., et al. "Current concepts in organic acidurias: understanding intra- and extracerebral disease manifestation". Journal of Inherited Metabolic Disease 36.4 (2013): 635-644.

10. El-Hattab AW., et al. "Molecular and clinical spectra of FBXL4 deficiency". Human Mutation 38.12 (2017): 1649-1659.

11. Gai X., et al. "Mutations in FBXL4, encoding a mitochondrial protein, cause early-onset mitochondrial encephalomyopathy". American Journal of Human Genetics 93.3 (2013): 482495.

12. Bonnen PE., et al. "Mutations in FBXL4 cause mitochondrial encephalopathy and a disorder of mitochondrial DNA maintenance". American Journal of Human Genetics 93.3 (2013): 471-481.

13. Huemer M., et al. "Clinical, morphological, biochemical, imaging and outcome parameters in 21 individuals with mitochondrial maintenance defect related to FBXL4 mutations". Journal of Inherited Metabolic Disease 38.5 (2015): 905-914.
14. Steward CG., et al. "Neutropenia in Barth syndrome: characteristics, risks, and management". Current Opinion on Hematology 26.1 (2019): 6-15.

15. Clarke SLN., et al. "Barth syndrome". Orphanet Journal of Rare Diseases 8 (2013): 23.

16. Schiff M., et al. "Clinical, laboratory and molecular findings and long-term follow-up data in 96 French patients with PMM2CDG (phosphomannomutase 2-congenital disorder of glycosylation) and review of the literature". Journal of Medical Genetics 54.12 (2017): 843-851.

\section{Assets from publication with us}

- Prompt Acknowledgement after receiving the article

- Thorough Double blinded peer review

- Rapid Publication

- Issue of Publication Certificate

- High visibility of your Published work

Website: www.actascientific.com/

Submit Article: www.actascientific.com/submission.php

Email us: editor@actascientific.com

Contact us: +919182824667 